\title{
Die Valenzisomerisierung des 9.10-Dewarisomers zum 9-t-Butylanthrazen
}

\author{
Burkhardt Jahn und Herbert Dreeskamp \\ Lehrstuhl B für Physikalische Chemie der TU Braunschweig \\ Z. Naturforsch. 37a, 376-379 (1982); eingegangen am 23. Februar 1982 \\ Valence Isomerization of the 9,10-Dewar-Isomer to 9-t-Butylanthracene
}

By DTA calorimetry the enthalpy of valence isomerization of 1-t-butyl-dibenzo-2,5-bicyclo$(2,2,0)$-hexadiene (9-t-butyl-9,10-Dewar-anthracene) 2 to 9 -t-butylanthracene 1 has been determined as $-172 \pm 5 \mathrm{~kJ} \mathrm{~mol}^{-1}$ in decaline at $350 \mathrm{~K}$. Photochemically the isomerization $2 \rightarrow 1$ occurs with the high quantum yield of $\Phi_{\mathrm{R}} \cong 0.4$ in contrast to the low quantum yield of the reverse photo-isomerization $\mathbf{1} \rightarrow \mathbf{2}$ of $\Phi_{\mathrm{R}}=0.016$ [5]. Since other meso-substituted anthracenes, e.g. 9-methyl-anthracene or 9-trimethylsilyl-anthracene do not photoreact to Dewar-isomers, it is concluded that a deformation of the anthracene sceleton is a prerequisite for this type of valence isomerization.

Die Photodimerisierung von Anthrazen und substituierten Anthrazenen in inerten Lösungsmitteln bei relativ hoher Konzentration $\left(>10^{-3} \mathrm{M}\right)$ ist eine der gut untersuchten photochemischen Reaktionen [1]. Sie ist begleitet von einer Selbstlöschung der Fluoreszenz, die im allgemeinen ein stark strukturiertes Spektrum besitzt [2]. Demgegenüber sind Anthrazene in inerten sauerstofffreien Lösungen bei niedriger Konzentration $\left(<10^{-5} \mathrm{M}\right)$ photostabil. Überraschenderweise wurde ein hiervon stark abweichendes Verhalten bei 9-t-Butylanthrazen 1 gefunden $[3,4,5]$. Unter den oben angegebenen Bedingungen ist nämlich das Absorptionsspektrum schwach strukturiert, das Fluoreszenzspektrum jedoch völlig unstrukturiert, und die Fluoreszenzquantenausbeute wie auch die Fluoreszenzlebensdauer klein $\left(\Phi_{\mathrm{F}} \cong 0,01, \tau_{\mathrm{F}} \cong 0,1 \mathrm{~ns}\right)$.

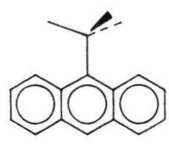

1

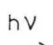

$\stackrel{\rightleftharpoons}{\rightleftharpoons, h v}$

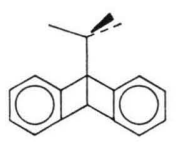

$\underline{\underline{2}}$
Während andere Anthrazene photochemisch stabil sind, erfolgt in inerten fluiden Lösungsmitteln eine photochemische Valenzisomerisierung von 9-tButylanthrazen 1 zu 9-t-Butyldewaranthrazen 2 (1-t-Butyldibenzo-2,5-bicyclo-(2,2,0)-hexadien) mit

Reprint requests to Prof. Dr. H. Dreeskamp, Lehrstuhl B für Physikalische Chemie der Technischen Universität Braunschweig, Hans-Sommer-Straße 10, D-3300 Braunschweig. der relativ hohen Quantenausbeute von $\Phi_{\mathrm{R}}=0,016$. Aus $\mathbf{2}$ wird in einer thermischen Reaktion $\mathbf{1}$ zurückgebildet, die mit einer hohen Aktivierungsenthalpie von $\Delta H^{\ddagger}=93 \mathrm{~kJ} \mathrm{~mol}^{-1}$ verläuft [5]. Bislang waren thermoreversible photochemische Valenzisomerisierungen von Aromaten zu Dewararomaten für substituierte Benzole [6,7] und substituierte Naphthaline [8] bekannt. Zur weiteren Klärung der Energetik dieser thermoreversiblen Photoreaktion haben wir zunächst die Reaktionsenthalpie der Rearomatisierungsreaktion $\mathbf{2} \rightarrow \mathbf{1}$ bestimmt.

Die Darstellung von 2 erfolgte photochemisch aus 1 , indem $40 \mathrm{mg}$ von 1 in $600 \mathrm{ml}$ spektroskopisch reinem Isopentan bei $-15^{\circ} \mathrm{C}$ für $4 \mathrm{~h}$ in einem Tauchbrennreaktor (Hg Hochdruckstrahler TQ 150, Fa. Heraeus, Hanau) belichtet wurde. Der Nachweis eines vollständigen Umsatzes von $\mathbf{1} \rightarrow \mathbf{2}$ $(>99,9 \%)$ erfolgte UV-spektroskopisch. Nach Abzug des Lösungsmittels in der Kälte wurden die verbleibenden farblosen Kristalle von $\mathbf{2}$ in der Kälte $\left(-15^{\circ} \mathrm{C}\right)$ in Decalin oder 1,3-Dichlorbenzol gelöst. $30 \mu \mathrm{l}$ der $0,5 \mathrm{M}$ Lösung wurden in DTAKapseln gefüllt, die kaltverschweißt verschlossen wurden. Die kalorischen Messungen wurden nach dem Prinzip der Differentialthermoanalyse mit dem Thermoanalysesystem TA 500 der Fa. Heraeus durchgeführt. Benutzt wurden konstante Heizraten zwischen 5 und $10 \mathrm{~K} \mathrm{~min}^{-1}$ von $170 \mathrm{~K}$ bis 380 K. Kapseln mit reinem Lösungsmittel dienten als Referenz und die Schmelzenthalpie von Naphthalin $\left(19 \mathrm{~kJ} \mathrm{~mol}^{-1}\right)$ zur Kalibrierung des Gerätes. Aus den experimentellen Registrierkurven von $\Delta T$ 
gegen die Zeit wurden sowohl die Reaktionsenthalpie $\Delta H$ wie auch die kinetischen Parameter $E_{\mathrm{a}}, \lg a, \Delta H^{\ddagger}$ und $\Delta S^{\neq}$nach der Methode von Borchardt und Daniels [9] mit dem von Wolf und Cammenga [10] modifizierten Verfahren bestimmt. Den Nachweis mit diesen Messungen ohne systematische Fehler der Rearomatisierung von $\mathbf{2} \rightarrow \mathbf{1}$ gemessen zu haben, erbringen die folgenden Beobachtungen:

a) Eine Reaktionsenthalpie wird nur beobachtet beim ersten Durchlauf einer DTA-Messung.

b) Die aus den DTA-Messungen bestimmten kinetischen Parameter sind identisch mit den UVspektroskopisch bestimmten Daten.

c) Nach Beendigung der Messung läßt sich UVspektroskopisch in der verwendeten Lösung kein 9-t-Butyldewaranthrazen 2 mehr nachweisen.

Die hier bestimmten kalorischen und kinetischen Daten für die Umwandlung von $\mathbf{2} \rightarrow \mathbf{1}$ sind in Tab. 1 angegeben, wie auch die früher [5] auf anderem Wege bestimmten kinetischen Daten für diese Reaktion und einige Daten aus der Literatur für die thermischen Rearomatisierungsreaktionen von Dewarbenzol [6,11-13], Dewarnapthalin [14] und 1,4-Dewaranthrazen [15]. Die einheitlich hohe Aktivierungsschwelle für diese Reaktionen ist in Übereinstimmung mit der Regel von Woodward und Hoffmann, mit der diese thermische Reaktion orbital - verboten ist [16]. Interessanterweise er- gibt sich jedoch - siehe Tabelle 1 - eine deutliche Verringerung des Betrages der Reaktionsenthalpie $\Delta H$ für die Umlagerung von 9-t-Butyl-9,10-dewaranthrazen 2 zu 9-t-Butylanthrazen 1 gegenüber der Umlagerung von Dewarbenzol zu Benzol. Dieser Unterschied ist verständlich, da im ersten Fall nur die Differenz der Resonanzenergie von Anthrazen zu der zweier Benzolringe, im zweiten Fall jedoch die volle Resonanzenergie eines Benzolringes gewonnen wird. Nimmt man die von Dewar nach dem Verfahren von Pople berechneten Werte der Resonanzenergien [17], so ergibt sich sogar eine ungefähre quantitative Übereinstimmung, worauf zuerst Pritchins und Grimme hingewiesen haben [18]. Ob aus dieser Übereinstimmung zu schließen ist, daß die Enthalpiedifferenz zwischen Aromat und Dewararomat allein durch den Unterschied in der Resonanzenergie bedingt sei, ist fraglich. Dieses würde bedeuten, daß die Werte der Resonanzenergien von Aromaten auf Dewararomaten übertragbar seien und die durch Änderung der Molekülgeometrie bedingten Enthalpieänderungen vernachlässigbar wären.

Bislang ist eine photochemische Valenzisomerisierung mit einer Quantenausbeute der Größenordnung von 0,01 bei meso-substituierten Anthrazenen allein bei 1 beobachtet worden. Beobachtungen an Molekülmodellen legen die Vermutung nahe, daß die t-Butylgruppe durch sterische Hinderung eine nennenswerte Deformation der Planarität des

Tab. 1. Reaktionsenthalpie und Kinetische Parameter der Isomerisierungsreaktion. Dewararomat $\rightarrow$ Aromat. a) Diese Arbeit; b) Keine Angabe; c) Ohne Lösungsmittel.

\begin{tabular}{|c|c|c|c|c|c|c|c|}
\hline Reaktion & $\begin{array}{l}\text { Lösungs- } \\
\text { mittel }\end{array}$ & $\begin{array}{l}\Delta H \\
{\left[\mathrm{~kJ} \cdot \mathrm{mol}^{-1}\right]}\end{array}$ & $\begin{array}{l}E_{\mathrm{a}} \\
{\left[\mathrm{kJ} \cdot \mathrm{mol}^{-1}\right]}\end{array}$ & $\begin{array}{l}\lg a \\
{\left[a \text { in } \sec ^{-1}\right]}\end{array}$ & $\begin{array}{l}\Delta H^{\neq} \\
{\left[\mathrm{kJ} \cdot \mathrm{mol}^{-1}\right]}\end{array}$ & $\Delta S^{\neq}$ & Lit. \\
\hline $\begin{array}{l}\text { Dewarbenzol } \rightarrow \\
\text { Benzol }\end{array}$ & $\begin{array}{l}\text { b) } \\
\text { Tetrachlor- } \\
\text { ethylen }\end{array}$ & $-251,0$ & & & $96,2 \pm 8,4$ & $-20,9 \pm 14,6$ & $\begin{array}{l}{[6]} \\
{[11]}\end{array}$ \\
\hline $\begin{array}{l}\text { Hexamethyldewar- } \\
\text { benzol } \rightarrow \\
\text { Hexamethylbenzol }\end{array}$ & b) & $\begin{array}{l}-249,0 \\
-235,1 \pm 2,5\end{array}$ & 155,7 & 15,03 & $\begin{array}{l}152,2 \\
151,9 \pm 1,7\end{array}$ & $\begin{array}{l}31,4 \\
32,6 \pm 2,5\end{array}$ & {$[12]$} \\
\hline $\begin{array}{l}\text { Dewarnaphthalin } \rightarrow \\
\text { Napthalin }\end{array}$ & $\begin{array}{l}\text { Squalan/ } \\
\text { n-Heptan }\end{array}$ & $-248,4 \pm 8$ & $99,1 \pm 2,6$ & $12,3 \pm 0,4$ & $96,3 \pm 2,5$ & $-18,4 \pm 7,7$ & {$[14]$} \\
\hline $\begin{array}{l}\text { 1,4-Dewaranthrazen } \rightarrow \\
\text { Anthrazen }\end{array}$ & n-Heptan & $\begin{array}{l}-313,8 \\
-334,7\end{array}$ & 110,9 & & & & {$[15]$} \\
\hline $\begin{array}{l}\text { 9-t-Butyldewar- } \\
\text { anthrazen } \rightarrow\end{array}$ & $\begin{array}{l}\text { Decalin } \\
\text { 1,3-Dichlor- } \\
\text { benzol }\end{array}$ & $-172,3 \pm 5,7$ & $94,8 \pm 3$ & $11,9 \pm 0,6$ & $92,3 \pm 3$ & $-25,4 \pm 8$ & [a] \\
\hline 9-t-Butylanthrazen & $\begin{array}{l}\text { n-Heptan } \\
\text { Äthanol }\end{array}$ & & $\begin{array}{l}93,3 \pm 2,9 \\
96,2 \pm 2,9\end{array}$ & $\begin{array}{l}11,9 \pm \\
12,5 \pm\end{array}$ & $\begin{array}{l}90,8 \pm 2,9 \\
93,7 \pm 2,9\end{array}$ & $\begin{array}{l}-25,1 \pm 8,4 \\
-17,2 \pm 8,4\end{array}$ & $\begin{array}{l}{[5]} \\
{[5]}\end{array}$ \\
\hline
\end{tabular}


Anthrazengerüstes schon im Grundzustand bewirkt. Der durch Lichtabsorption erreichte FranckCondon-Zustand von $\mathbf{1}$ wäre damit auch in die Richtung auf die Geometrie des Dewaranthrazens verzerrt und eine Isomerisierung zu 2 leichter möglich. Zwei Beobachtungen stützen diese Vorstellung:

(a) Wie für zahlreiche meso-substituierte Anthrazene, z.B. 9-Methylanthrazen [1] ist auch für 9-Trimethylsilylanthrazen 3 die Photodimerisierung bekannt [19]. Bei sorgfältigen Untersuchungen dieser Substanz in inerten Lösungsmitteln (z. B. Isopentan) bei niedriger Konzentration $\left(<10^{-5} \mathrm{M}\right)$ und tiefer Temperatur $(230 \mathrm{~K})$ fanden wir kein Anzeichen für eine Umlagerung zum Dewarisomer. Falls die photochemische Valenzisomerisierung von 3 überhaupt erfolgt, ist ihre Quantenausbeute $\Phi_{\mathrm{R}}<10^{-4}$. Wegen des größeren C-Si-Abstandes $\left(R_{\mathrm{CC}} \cong 1,54 \AA, R_{\mathrm{SiC}} \cong 1,94 \AA\right)[20]$ wird die durch den Substituenten bewirkte sterische Verzerrung des Anthrazengerüstes in $\mathbf{3}$ wesentlich geringer sein als in 1. Dementsprechend ist in $\mathbf{3}$ die Fluoreszenzquantenausbeute hoch, die Schwingungsstruktur des Fluoreszenzspektrums erkennbar und die Tendenz zur Valenzisomerisierung klein.

b) Bei drastischer Erhöhung der Viskosität des Lösungsmittels sinkt die Quantenausbeute $\Phi_{\mathrm{R}}$ der Photoreaktion $\mathbf{1} \rightarrow \mathbf{2}$, die Quantenausbeute der Fluoreszenz $\Phi_{\mathrm{F}}$ von 1 steigt an, und das Fluoreszenzspektrum von $\mathbf{1}$ wird stark strukturiert. Typische Werte der Quantenausbeute von 1 sind: Lösungsmittel Paraffin (Uvasol, Merck), $\Phi_{\mathrm{R}}=0,016$ und $\Phi_{\mathrm{F}}=0,07$ bei $300 \mathrm{~K}$ und $\Phi_{\mathrm{R}}=0,010$ und $\Phi_{\mathrm{F}}=0,20$ bei $270 \mathrm{~K}$. Lösungsmittel 2,2-Dimethylbutan und n-Pentan $(8: 3), \Phi_{\mathrm{R}}=0,016$ und $\Phi_{\mathrm{F}}=$ 0,01 bei $300 \mathrm{~K}$ und $\Phi_{\mathrm{R}}=0,015$ und $\Phi_{\mathrm{F}}=0,02$ bei $270 \mathrm{~K}$.

Bei Anregung von $\mathbf{2}$ in die langwelligste strukturierte Absorptionsbande bei $280 \mathrm{~nm}$ beobachtet

[1] Siehe z.B.: D. O. Cowan u. R. L. Drisko, Elements of Organic Photochemistry, Plenum Press, New York 1976 , pp. 37.

[2] Siehe z.B.: Th. Förster, Fluoreszenz organischer Verbindungen. Vandenhoeck und Ruprecht, Göttingen 1951, pp. 230.

[3] H. Dreeskamp und J. Pabst, Kurzfassung ,66. Vortragstagung der Fachgruppe Photochemie der GDCh", Göttingen, 21.-23. 11. 1979.

[4] H. Güsten, M. Mintas und L. Klasinc, J. Amer. Chem. Soc. 102, 7936 (1980).

[5] H. Dreeskamp, B. Jahn u. J. Pabst, Z. Naturforsch. 36 a, 665 (1981). man eine Fluoreszenz von $\mathbf{2}$ und eine Valenzisomerisierung von $\mathbf{2} \rightarrow \mathbf{1}$ mit hohen Quantenausbeuten $\left(\Phi_{\mathrm{R}} \cong 0,4\right)$. Während bei 2 die Summe der Quantenausbeuten dieser beiden Prozesse nahe 1 ist, beträgt die Summe der analogen Quantenausbeuten bei 1 nur $\cong 0,03$.

Aus der experimentell gefundenen Ähnlichkeit der thermodynamischen und kinetischen Werte $\Delta H$, $\lg a$ und $E_{\mathrm{a}}$ der Valenzisomerisierung von Dewararomaten $\mathrm{zu}$ Aromaten ist $\mathrm{zu}$ schließen, daß das Korrelationsdiagramm für die Isomerisierung Benzol zu Dewarbenzol [6,21] qualitativ auch bei Anthrazenen, speziell für die Umwandlung $1 \mathrm{zu} 2$ gültig ist. Damit wäre die Deutung der beobachteten Valenzisomerisierung und strahlungslosen Desaktivierung von 1 wie folgt möglich: Nach Anregung von 1 wird das Molekül vom zunächst erreichten Franck-Condon-Zustand 1* aus längs der Potentialfläche des Anregungszustandes auf die gewinkelte Geometrie des Dewarisomers hin verzerrt. Dabei wird die Energiedifferenz zwischen Anregungszustand und Grundzustand kleiner, und ein Übergang zur Potentialfläche des Grundzustandes wird möglich. Von dem so erreichten Punkt auf der Potentialfläche des Grundzustandes aus erfolgt eine Relaxation zur Ausgangsverbindung 1 mit höherer Häufigkeit als zum Valenzisomer 2. Strahlungslose Desaktivierung von $1 *$ und photochemische Valenzisomerisierung von 1 zu 2 wären nach dieser Deutung jedoch nur möglich - wie experimentell beobachtet - bei hinreichender Fluidität des Lösungsmittels.

Diese Arbeit wurde durch Mittel des Fonds der Chemie und Forschungsförderungsmittel des Landes Niedersachsen unterstützt. Unser besonderer Dank gilt unseren Kollegen H. K. Cammenga und J. Reichelt, die die kalorischen Messungen in ihrem thermochemischen Labor ermöglichten.

[6] N. J. Turro, Modern Molecular Photochemistry, Benjamin Publ. Co., Inc., Menlo Park 1978, p. 502.

[7] E. E. van Tamelen u. S. P. Pappas, J. Amer. Chem. Soc. 84, 3789 (1962).

[8] W. L. Mandella u. R. W. Franck, J. Amer. Chem. Soc. 95, 971 (1973).

[9] H. J. Borchardt u. F. Daniels, J. Amer. Chem. Soc. 79, 41 (1957).

[10] E. Wolf u. H. K. Cammenga, Z. Phys. Chemie, N. F. 107, 21 (1977).

[11] R. Breslow, J. Napierski u. A. H. Schmidt, J. Amer. Chem. Soc. 94, 5906 (1972).

[12] F. W. Oth, Angew. Chemie 80, 633 (1968). 
[13] W. Adam u. J. C. Chang, Intern. J. Chem. Kinetics 1, 487 (1969).

[14] J. W. Grimme u. U. Heinze, Chem. Ber. 111, 2563 (1978).

[15] N. C. Yang, R. C. Carr, E. Li, J. K. McVey u. S. A. Rice J. Amer. Chem. Soc. 96, 2297 (1974).

[16] R. B. Woodward u. R. Hoffmann, die Erhaltung der Orbitalsymmetrie, Verlag Chemie, Weinheim 1970.
[17] M. J. S. Dewar, The Molecular Orbital Theory of Organic Chemistry, McGraw Hill, New York 1969, p. 177.

[18] W. Pritschins u. W. Grimme, Tetrahedron Letters 47, 4545 (1979) und private Mitteilung W. Grimme.

[19] A. Castellan, J.-P. Desvergne u. H. Bouas-Laurant, Nouveau J. de Chim. 3, 231 (1979).

[20] F. A. Cotton u. G. Wilkinson, Anorganische Chemie, Verlag Chemie, Weinheim 1967 p. 97.

[21] I. Haller, J. Chem. Phys. 47, 1117 (1967). 